\title{
Tapentadol: an effective option for the treatment of back pain
}

This article was published in the following Dove Medical Press journal: Journal of Pain Research

\section{Flaminia Coluzzi' \\ Enrico Polati ${ }^{2}$ \\ Ulderico Freo ${ }^{3}$ \\ Mariagrazia Grilli ${ }^{4}$}

'Department of Medical and Surgical Sciences and Biotechnologies, Unit of Anesthesia, Intensive Care and Pain Medicine, SAPIENZA University of Rome - Polo Pontino, Latina, Italy; ${ }^{2}$ Anesthesia and Intensive Care, Pain Relief Center, Ospedale Policlinico GB Rossi, Verona, Italy; ${ }^{3}$ Anesthesiology and Intensive Medicine, Department of Medicine DIMED University of Padua, 35100 Padua, Italy; ${ }^{4}$ Laboratory of Neuroplasticity, Department of Pharmaceutical Sciences, University of Piemonte Orientale, 28100 Novara, Italy
Correspondence: Flaminia Coluzzi Department of Medical and Surgical Sciences and Biotechnologies, Unit of Anesthesia, Intensive Care and Pain Medicine, SAPIENZA University of Rome - Polo Pontino, Corso della Repubblica 79, 04100 Latina, Italy

Tel +390773 65II

Email flaminia.coluzzi@uniromal.it

\begin{abstract}
Back pain, including low back pain and neck pain, is the leading cause of disability worldwide. This type of pain is challenging to treat, since it presents both a nociceptive and a neuropathic component. The latter also contributes to the evolution of pain toward chronification. Treatment selection should therefore consider the ability to prevent this event. Tapentadol is characterized by a unique and innovative peculiar mechanism of action that makes it the first representative of a new class of central strong analgesics referred to as MOR-NRI. This molecule acts both on the nociceptive and neuropathic components of pain, and it can therefore be effective in the treatment of a mixed pain condition such as back pain. This narrative review discusses the rationale for the use of tapentadol in both low back pain and neck pain and presents available clinical data. Overall, data show that tapentadol prolonged release is a well-grounded treatment for chronic back pain, sustained by a strong mechanistic rationale and robust evidence. Given also the availability of long-term efficacy and safety data, we believe that this molecule should be considered as an elective therapy for chronic back pain.
\end{abstract}

Keywords: tapentadol, low back pain, neck pain

\section{Introduction}

Low back pain (LBP) is one of the most frequent chronic pain conditions worldwide, with a lifetime prevalence $>70 \%$ in western countries and a heavy burden for the healthcare system. ${ }^{1-3}$ Indeed, LBP is now considered the leading cause of disability worldwide. Remarkably, more than two out of three patients experiencing acute LBP attacks ultimately develop chronic LBP. ${ }^{4}$ Moreover, chronic LBP is frequently associated with comorbid conditions, including depression, panic and anxiety disorders, and sleep disturbances. ${ }^{5}$

Although sometimes neglected when compared with LBP, neck pain is also a common disabling disease. ${ }^{6}$ Indeed, the prevalence of neck pain can be as high as $23 \%$. $^{7}$ Therefore, the economic burden of neck pain is also high, mostly due to increased need of medical visits, physiotherapy, pharmacological and surgical treatments, working days lost, and compensation expenditure. ${ }^{8}$

Noteworthy, chronic LBP or neck pain - collectively, back pain - results from chronification processes occurring over time and involving plastic alterations of the involved structures. ${ }^{9-11}$ Back pain presents in the wide majority of cases $(>90 \%)$ as a neuropathic component. ${ }^{9,12}$ Proper selection of treatment is therefore of paramount importance. In this setting, routine use of classical opioids is not recommended, since benefits are small and substantial risks exist, including overdose and addiction potential, and poorer long-term outcomes than without use..$^{13}$ 
Tapentadol is a dual $\mu$-opioid receptor (MOR) agonist and noradrenaline reuptake inhibitor (NRI), which was rationally designed, and represents the first and unique member of a new class of analgesic agents, MOR-NRI.${ }^{14}$ Remarkably, the " $\mu$-load" of tapentadol is $\leq 40 \%$ relative to classical MOR agonists. This reduced $\mu$-load results from the combination and synergistic interaction of the two mechanisms of analgesic action. Due to this, lower opioid activity is needed to reach comparable analgesia and therefore a more favorable tolerability profile is achieved, in terms of gastrointestinal, respiratory, and endocrinological adverse events. ${ }^{15,16}$ Moreover, tapentadol shows minimal serotoninergic activity, with potential safety advantages over the long term in terms of risk of emesis. ${ }^{17}$

This narrative review discusses the rationale for the use of tapentadol in both LBP and neck pain and presents available clinical data.

\section{Tapentadol in the treatment of LBP Rationale of use}

Chronic LBP is a heterogeneous condition, where both nociceptive and neuropathic pain mechanisms may be involved. ${ }^{5}$ In particular, nociceptive pain results from the activation of nociceptors as a response to tissue injury and biomechanical stress. On the other hand, the neuropathic component arises from injury affecting the nerve roots that innervate the spine and lower limbs, and pathological invasive innervation of the damaged lumbar discs. This latter component has often been underestimated when selecting appropriate treatment for LBP. ${ }^{5}$

Indeed, several practice guidelines for the management of chronic LBP have been published. ${ }^{18-21}$ In most cases, they advise a multimodal approach for the management of chronic LBP, combining pharmacological therapies with nonpharmacological approaches. However, these guidelines typically do not include specific recommendations for the treatment of the neuropathic components of this type of pain. Moreover, available guidelines for the treatment of neuropathic pain are usually focused on disease other than LBP, such as postherpetic neuralgia or painful diabetic neuropathy. ${ }^{22-25}$

Noteworthy, studies of LBP are typically short term $(<3$ months duration), and evidence of effectiveness and safety associated with long-term treatment is currently limited. ${ }^{5,26}$ In addition, few head-to-head trials comparing different treatments and combination strategies have been published, and therefore, direct comparisons of drug efficacy and tolerability are not possible.
Tapentadol prolonged release (PR) has been proven to provide a strong analgesic effect, due to its synergic MOR and NRI action. ${ }^{9,10,27,28}$ Remarkably, the different pharmacological effects of tapentadol are not synergic in terms of adverse effects. ${ }^{29}$ Noteworthy, these benefits are paralleled by improvements in quality of life (QoL).$^{30}$ Therefore, tapentadol PR may be considered a particularly suitable option in patients with chronic LBP, given the important neuropathic component of this condition. The efficacy of tapentadol in this setting is also supported by the results of a Cochrane Review - although published in 2015, it also includes studies on osteoarthritis - which shows that tapentadol PR is associated with a reduction in pain intensity compared with placebo and oxycodone and presents improved safety compared with oxycodone ${ }^{31}$ However, the authors of this review pointed out that some methodological flaws were present in the studies considered, therefore reducing the quality of the results.

\section{Clinical data}

The efficacy and safety of tapentadol PR were extensively tested in patients with LBP, both in an experimental and in a "field-practice" settings, and in comparative studies (Table 1).

In the pivotal trial of tapentadol in LBP, with a randomized, double-blind, placebo-controlled design, $\sim 1,000$ patients were assigned to tapentadol PR 100-250 mg twice daily, oxycodone controlled release (CR) 20-50 mg twice daily or placebo over 15 weeks (3-week titration period, 12-week maintenance period). ${ }^{32}$ Overall, both tapentadol PR and oxycodone significantly reduced average pain intensity, as assessed by the numerical rating scale (NRS), vs placebo at week 12 and throughout the maintenance period; however, tapentadol was associated with a lower incidence of treatment-emergent adverse events (TEAEs). Indeed, the incidence of gastrointestinal TEAEs, including constipation, nausea, and vomiting, was $43.7 \%$ with tapentadol and $61.9 \%$ with oxycodone CR. Moreover, the odds of experiencing constipation or the composite of nausea and/or vomiting were lower with tapentadol PR than with oxycodone CR $(P<0.001)$.

In another Phase IIIb study, with an open-label design and without a control group, Gálvez et al evaluated the effectiveness and tolerability of tapentadol PR in the management of severe chronic LBP in patients with poor tolerance to opioids. ${ }^{33}$ Equi-analgesic ratios for tapentadol to strong opioids were calculated, and patients switched directly to tapentadol. Patients received tapentadol PR 50-250 mg twice daily over a 5 -week titration and a 7 -week maintenance 







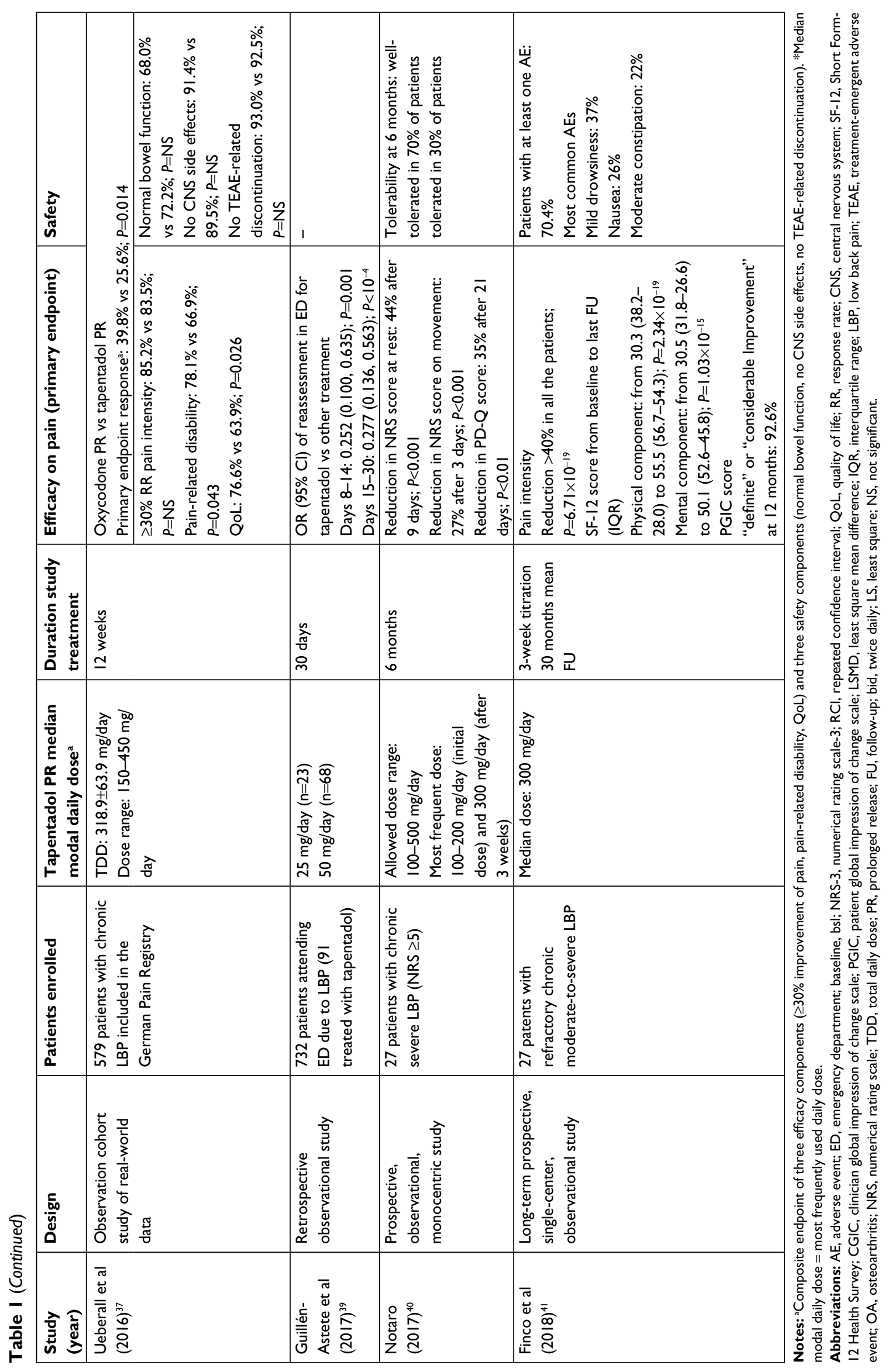


period. Responder rate (ie, reduced intensity of pain by NRS) at week 6 was $80.9 \%(P<0.0001$ vs the null responder hypothesis rate, $<60 \%$ ), resulting in a positive trial despite premature termination. Moreover, reduced intensity of pain was maintained up to week 12 . The prevalence of adverse events was reported as the reason for switching to tapentadol - in most cases constipation and nausea - decreased over time. Overall, these data show that tapentadol PR provided at least comparable analgesia and improved tolerability vs strong opioids in patients with severe, chronic LBP. In 2015 , Baron et al published the results of a randomized, doubleblind study comparing the effectiveness and tolerability of tapentadol PR monotherapy vs tapentadol PR/pregabalin combination therapy for severe chronic LBP with a neuropathic component. ${ }^{34}$ All patients had painDETECT "unclear" or "positive" ratings and average pain intensity at baseline of $\geq 6$ on an NRS. Patients were then titrated to tapentadol PR $300 \mathrm{mg}$ /day over 3 weeks, and those with $\geq 1$-point decrease in pain intensity and average pain intensity $\geq 4$ were assigned to tapentadol PR (500 mg/day) or tapentadol PR (300 mg/ day)/pregabalin (300 mg/day) for an 8-week comparative period. In the per-protocol population, which consisted of 288 patients, the effectiveness of tapentadol PR was comparable to tapentadol PR/pregabalin; similarly, neuropathic pain and QoL measures improved in both the groups. Tolerability was good, but the incidence of the composite of dizziness and/or somnolence was lower with tapentadol PR (16.9\%) compared with tapentadol PR/pregabalin $(27.0 \% ; P=0.0302)$. According to these data, tapentadol PR monotherapy may be considered a favorable treatment option for severe LBP with a neuropathic component. In the extension phase of this trial, a subpopulation with pain intensity $<4$ continued receiving tapentadol PR $300 \mathrm{mg}$ /day during an 8-week, openlabel continuation arm. ${ }^{35}$ Overall, greater improvements in all measures were observed for this selected population. In another randomized, controlled, open-label, Phase IIIb/IV study by the same group, the effectiveness of tapentadol PR was compared with that of oxycodone/naloxone PR in opioidnaive patients with severe chronic LBP and a neuropathic pain component. ${ }^{36}$ Patients were randomly assigned to tapentadol PR $50 \mathrm{mg}$ twice daily or oxycodone/naloxone PR $10 \mathrm{mg} / 5$ $\mathrm{mg}$. After a 21-day titration, maximum allowed twice-daily doses were $250 \mathrm{mg}$ for tapentadol and $40 \mathrm{mg} / 20 \mathrm{mg}$ for oxycodone/naloxone. Target doses were then continued for 9 weeks. The primary effectiveness endpoint was the change in NRS-3 from baseline to final evaluation; the exact repeated confidence interval for tapentadol PR minus oxycodone/ naloxone PR was used to establish noninferiority (upper limit <1.3) and superiority (confirmatory analyses). For the primary effectiveness endpoint (change in pain intensity from baseline to final evaluation), tapentadol PR was superior over oxycodone/naloxone $(P<0.003) .{ }^{36}$ Pain intensity at baseline was 7.6 in both groups; at final evaluation, it was 4.8 with oxycodone/naloxone (mean change vs baseline, -2.7 ) and 3.9 with tapentadol PR (mean change vs baseline, -3.7 ). ${ }^{36}$ Moreover, improvements in painDETECT and Neuropathic Pain Symptom Inventory scores were greater with tapentadol $\mathrm{PR}$, and this molecule was associated with a more favorable tolerability profile. On these bases, the authors concluded that tapentadol PR may be considered a first-line option for managing severe chronic LBP with a neuropathic pain component.

Several studies have also investigated the effectiveness of tapentadol PR for the treatment of LBP in the "fieldpractice" setting, although with all the inherent limitations of any observational analysis. Ueberall et al analyzed randomly selected data of the German Pain Registry, collected over a 12-week period, of adult patients treated with either tapentadol PR ( $n=133)$ or oxycodone/naloxone $(n=128) .{ }^{37}$ The primary endpoint was a composite of $\geq 30 \%$ improvement of pain, pain-related disability, and QoL and three tolerability components (normal bowel function, absence of either central nervous system side effects, and TEAE-related treatment discontinuation during the observation period). Overall, the two treatments were comparable in terms of effectiveness and safety. However, this study was judged to be affected by major methodological bias, ${ }^{38}$ and therefore, its results should be considered with caution. A retrospective observational study was conducted by Guellen-Astete et al, who evaluated patients attending the emergency department (ED) due to LBP over a period of 24 months. ${ }^{39}$ Among 732 patients referring to the ED, 91 were treated with tapentadol. In the first month after the first assessment, reassessments were less frequently observed in the tapentadol group, reaching statistical significance from day 8 onwards. Patients on tapentadol also had a better clinical evolution of pain compared with those who did not receive this analgesic drug. In another 6-month "fieldpractice" monocentric experience, with a prospective design, Notaro evaluated 27 patients treated with tapentadol PR for chronic ( $>1$ year in about $90 \%$ of patients) severe LBP. ${ }^{40}$ All patients had received previous analgesic therapy; treatment with tapentadol PR was started at $100 \mathrm{mg} /$ day and could be increased up to $500 \mathrm{mg}$ /day. Tapentadol PR promptly reduced the average intensity of pain at rest ( $-44 \%$ at 9 days) and pain on movement ( $-27 \%$ at 3 days; $P<0.01$ for both comparisons). Moreover, the neuropathic component of pain, QoL, 
and health status improved. No relevant safety signals were reported. In a long-term prospective experience, Finco et al investigated the effectiveness, safety, and tolerability of oral formulation of tapentadol PR in 27 patients with refractory chronic LBP over a long-term follow-up (up to 51 months). ${ }^{41}$ All the patients reported a significant improvement of pain intensity and QoL at last follow-up, and, remarkably, no relevant safety concerns were reported. Overall, these results further confirm the long-term effectiveness, safety, and tolerability of oral tapentadol PR for the treatment of chronic LBP.

The identification of predictive factors of response would improve treatment selection and reduce healthcare costs. In a retrospective analysis of an open-label study, 46 baseline characteristics of the 122 evaluated patients were included in statistical prediction modeling. ${ }^{42}$ Overall, demographic data were not relevant for response prediction. The most important predictive factors were QoL and functionality. Neuropathic symptoms (high painDETECT score) had a positive predictive validity, while painful attacks and classical yellow flags (depression, anxiety) were negative predictors of response.

A remarkable new field of investigation is represented by the association of nutraceuticals and tapentadol in the treatment of $\mathrm{LBP}^{43}$ In a pilot Italian study, the addition of ultra-micronized palmitoylethanolamide to tapentadol led to a major reduction in pain intensity, with a good QoL and without any serious adverse event. ${ }^{43}$

\section{Tapentadol in the treatment of neck pain}

Neck pain may be caused by several different conditions that compress, irritate, and eventually destroy sensitive structures, including the annulus fibrosus, posterior longitudinal ligament, and the capsule of the zygapophyseal joints. ${ }^{44}$ Remarkably, relapses and establishment of chronic pain are frequent among patients with neck pain and cervical radiculopathy, leading to major impairments in QoL, activity limitation, and disability. Central sensitization is thought to play a major role in the progression to chronic neck pain. ${ }^{9}$ However, only few studies are available on the efficacy of different pharmacological treatments for neck pain, and the only published recommendations on the pharmacological approach for this disease is based on expert opinion. ${ }^{6}$

On these bases, tapentadol PR has been tested in the management of chronic neck pain, given its dual action on the nociceptive and neuropathic components of pain. ${ }^{6}$ In a small observational study, Billeci et al evaluated 54 patients with moderate-to-severe (mean NRS 8.1) chronic neck pain receiving tapentadol PR $100 \mathrm{mg} /$ day; dosage could be adjusted according to clinical needs. Over a 12-week period, the dosage of tapentadol PR increased up to $204.5 \pm 102.8$ $\mathrm{mg}$ /day. Mean pain intensity at movement decreased over time (mean change at final evaluation vs baseline, -5.9$){ }^{6}$ At baseline, $70 \%$ of patients presented a positive neuropathic component, and this percentage decreased to $23 \%$ at 12 weeks; a 35\% decrease was evident already at week $2 .^{6}$ Tapentadol PR was also associated with an improvement in the Neck Disability Index scores (from 55.6 \pm 18.6 at baseline to $19.7 \pm 20.9$ at 12 weeks; $P<0.01$ ), and with increased range of motion in all three planes, particularly in lateral flexion. QoL significantly improved, and $\sim 90 \%$ of patients rated their overall condition as much/very much improved. No patients discontinued tapentadol due to side effects and the use of other analgesics diminished during the observed period. Overall, these findings suggest that tapentadol PR is effective and well tolerated in patients with moderate-to-severe chronic neck pain and is associated with a relevant improvement of movement functionality and QoL.

\section{Conclusion}

Back pain, including LBP and neck pain, is the leading cause of disability worldwide. This type of pain is challenging to treat, since it presents, in the wide majority of cases, as a mixed pain characterized by a nociceptive and a neuropathic component. The latter also contributes to the chronification. Treatment selection should therefore consider the ability to prevent pain chronification by acting on the noradrenergic axis.

The pharmacological profile of tapentadol, combining synergistically MOR agonism and NRI in one molecule, appears to be unique, and therefore, this molecule has been proposed to be the first-in-class for a new class of centrally acting analgesics, namely MOR-NRI. ${ }^{14}$ Experimental evidence that NRI is a key mechanism that can be predominant in chronic LBP reinforces the concept that tapentadol is different from classical opioids and may therefore be an a priori choice for the treatment of chronic, neuropathic, and mixed pain. ${ }^{15,45,46}$

Clinical data on the efficacy of tapentadol PR in back pain are quite solid and do confirm its strong pharmacological rationale of use. Moreover, tapentadol PR is well-tolerated, and it is associated with a negligible incidence of adverse events associated with opioid therapy (eg, constipation and gastrointestinal events) and effects on hormonal axes. ${ }^{47}$ This favorable safety profile is of utmost importance, given the frequent need of long-term treatment in patients with back pain. To this end, it is worth mentioning that tapentadol has a low potential of abuse and can be easily titrated and tapered in clinical practice, ${ }^{48,49}$ 
dedicated studies should further investigate the titration/tapering and exit strategies with tapentadol PR.

In conclusion, tapentadol PR is a well-grounded treatment for chronic back pain, sustained by a strong mechanistic rationale and robust evidence. Given also the availability of long-term efficacy and safety data, we believe that this molecule should be considered as the elective therapy for chronic back pain.

\section{Key points}

- Back pain, including LBP and neck pain, is the leading cause of disability worldwide.

- This pain is challenging to treat, since it usually presents as a mixed pain characterized by a nociceptive and a neuropathic component. The latter also contributes to chronification.

- Treatment selection should therefore consider the ability to prevent chronification by acting on the noradrenergic axis.

- Tapentadol is characterized by a unique peculiar mechanism of action, and it is the only member of the MORNRI class of analgesic. This molecule acts on both the nociceptive and neuropathic components of pain, and it can be therefore effective in the treatment of back pain.

- Clinical data on the efficacy of tapentadol PR in back pain are quite solid and do confirm its strong pharmacological rationale of use.

- Tapentadol PR is well tolerated, and it is associated with a negligible incidence of adverse events associated with opioid therapy (eg, constipation and gastrointestinal events). This favorable safety profile is of utmost importance, given the frequent need of long-term treatment in patients with back pain.

\section{Acknowledgments}

Editorial assistance was provided by Luca Giacomelli, $\mathrm{PhD}$, Ambra Corti, and Aashni Shah. This assistance and fees for publications were supported by Grunenthal.

\section{Disclosure}

FC served as speaker for Grunenthal and received speaker's and/or consultancy fees from Grunenthal and Angelini. UF received consultancy fee from Grunenthal. The other authors report no conflicts of interest in this work.

\section{References}

1. Buchbinder R, van Tulder M, Öberg B, et al. Lancet low back pain series working group. Low back pain: a call for action. Lancet. 2018;6736(18):30488-30484.
2. Kaplan W, Wirtz VJ, Mantel-Teeuwisse A, Stolk P, Duthey B, Laing R Priority Medicines for Europe and the World: 2013 update. Geneva, Switzerland: World Health Organization; 2013. Available from: http:// www.who.int/medicines/areas/priority_medicines/MasterDocJune28_ FINAL_Web.pdf?ua=1. Accessed October 1, 2015.

3. Hartvigsen J, Hancock MJ, Kongsted A, et al. Lancet low back pain series working group. What low back pain is and why we need to pay attention. Lancet. 2018;6736(18):30480.

4. Itz CJ, Geurts JW, van Kleef M, Nelemans P. Clinical course of nonspecific low back pain: a systematic review of prospective cohort studies set in primary care. Eur J Pain. 2013;17(1):5-15.

5. Baron R, Binder A, Attal N, Casale R, Dickenson AH, Treede RD. Neuropathic low back pain in clinical practice. Eur J Pain. 2016;20(6):861-873.

6. Billeci D, Coluzzi F. Tapentadol extended release for the management of chronic neck pain. J Pain Res. 2017;10:495-505.

7. Hoy DG, Protani M, De R, Buchbinder R. The epidemiology of neck pain. Best Pract Res Clin Rheumatol. 2010;24(6):783-792.

8. Hogg-Johnson S, van der Velde G, Carroll LJ, et al. Bone and joint decade 2000-2010 Task Force on neck pain and its associated disorders. The burden and determinants of neck pain in the general population: results of the bone and joint decade 2000-2010 Task Force on neck pain and its associated disorders. Spine. 2008;33(4 Suppl): S39-51.

9. Coluzzi F, Fornasari D, Pergolizzi J, Romualdi P. From acute to chronic pain: tapentadol in the progressive stages of this disease entity. Eur Rev Med Pharmacol Sci. 2017;21(7):1672-1683.

10. Sánchez del Águila MJ, Schenk M, Kern KU, Drost T, Steigerwald I. Practical considerations for the use of tapentadol prolonged release for the management of severe chronic pain. Clin Ther. 2015;37(1): 94-113.

11. Morlion B, Coluzzi F, Aldington D, et al. Pain chronification: what should a non-pain medicine specialist know? Curr Med Res Opin. 2018;34(7):1169-1178.

12. Mehra M, Hill K, Nicholl D, Schadrack J. The burden of chronic low back pain with and without a neuropathic component: a healthcare resource use and cost analysis. J Med Econ. 2012;15(2): $245-252$.

13. Foster NE, Anema JR, Cherkin D, et al. Lancet low back pain series Working Group. Prevention and treatment of low back pain: evidence, challenges, and promising directions. Lancet. 2018;S0140-6736(18): 30489-6.

14. Kress HG. Tapentadol and its two mechanisms of action: is there a new pharmacological class of centrally-acting analgesics on the horizon? Eur J Pain. 2010;14(8):781-783.

15. Raffa RB, Elling C, Tzschentke TM. Does 'strong analgesic' equal 'strong opioid'? Tapentadol and the concept of ' $\mu$-load'. Adv Ther 2018; Epub ahead of print

16. Coluzzi F, Billeci D, Maggi M, Corona G. Testosterone deficiency in non-cancer opioid-treated patients. J Endocrinol Invest. 2018;41(12): 1377-1388.

17. Tzschentke TM, Folgering JH, Flik G, De Vry J. Tapentadol increases levels of noradrenaline in the rat spinal cord as measured by in vivo microdialysis. Neurosci Lett. 2012;507(2):151-155.

18. Chou R, Qaseem A, Snow V, et al. Clinical efficacy assessment Subcommittee of the American College of Physicians, American College of Physicians, American Pain Society low back pain guidelines panel. Diagnosis and treatment of low back pain: a joint clinical practice guideline from the American College of Physicians and the American Pain Society. Ann Intern Med. 2007;147:478-491.

19. National Institute for Health and Clinical Excellence. Early management of persistent non-specific low back pain; 2009. Available from: http:// www.nice.org.uk/guidance/CG88. Accessed October 1, 2015.

20. Koes BW, van Tulder M, Lin CW, Macedo LG, Mcauley J, Maher C. An updated overview of clinical guidelines for the management of non-specific low back pain in primary care. Eur Spine J. 2010;19(12): 2075-2094. 
21. German Medical Association, National Association of Statutory Health Insurance Physicians, and Association of Scientific Medical Societies. National disease management guideline: low back pain - short version 4; 2013. Available from: http://www.versorgungsleitlinien.de/themen/ kreuzschmerz/pdf/nvl-kreuzschmerz-kurz-engl-4.pdf. Accessed October $1,2015$.

22. Attal N, Cruccu G, Baron R, et al. EFNS guidelines on the pharmacological treatment of neuropathic pain: 2010 revision. Eur J Neurol. 2010;17(9):1113-e88.

23. Dworkin RH, O’Connor AB, Kent J, et al. Interventional management of neuropathic pain: NeuPSIG recommendations. Pain. 2013;154(11): 2249-2261.

24. National Institute for Health and Clinical Excellence. Neuropathic pain - pharmacological management: The pharmacological management of neuropathic pain in adults in non-specialist settings; 2013. Available from: http://www.nice.org.uk/guidance/cg173. Accessed October 1, 2015.

25. Finnerup NB, Attal N, Haroutounian S, et al. Pharmacotherapy for neuropathic pain in adults: a systematic review and meta-analysis. Lancet Neurol. 2015;14(2):162-173.

26. Mathieson S, Maher CG, Mclachlan AJ, et al. Trial of pregabalin for acute and chronic sciatica. $N$ Engl J Med. 2017;376(12):1111-1120.

27. Monti S, Caporali R. Chronic pain: the burden of disease and treatment innovations. Reumatismo. 2015;67(2):35-44.

28. Schwittay A, Schumann C, Litzenburger BC, Schwenke K. Tapentadol prolonged release for severe chronic pain: results of a noninterventional study involving general practitioners and internists. J Pain Palliat Care Pharmacother. 2013;27(3):225-234.

29. Cowan A, Raffa RB, Tallarida CS, et al. Lack of synergistic interaction between the two mechanisms of action of tapentadol in gastrointestinal transit. Eur J Pain. 2014;18(8):1148-1156.

30. Buynak R, Rappaport SA, Rod K, et al. Long-term safety and efficacy of tapentadol extended release following up to 2 years of treatment in patients with moderate to severe, chronic pain: results of an open-label extension trial. Clin Ther. 2015;37(11):2420-2438.

31. Santos J, Alarcão J, Fareleira F, Vaz-Carneiro A, Costa J. Tapentadol for chronic musculoskeletal pain in adults. Cochrane Database Syst Rev. 2015;5:CD009923.

32. Buynak R, Shapiro DY, Okamoto A, et al. Efficacy and safety of tapentadol extended release for the management of chronic low back pain: results of a prospective, randomized, double-blind, placeboand active-controlled phase III study. Expert Opin Pharmacother. 2010;11(11):1787-1804.

33. Gálvez R, Schäfer M, Hans G, Falke D, Steigerwald I. Tapentadol prolonged release versus strong opioids for severe, chronic low back pain: results of an open-label, phase 3B study. Adv Therapy. 2013;30(3): 229-259.

34. Baron R, Martin-Mola E, Müller M, Dubois C, Falke D, Steigerwald I. Effectiveness and safety of tapentadol prolonged release (PR) versus a combination of tapentadol PR and pregabalin for the management of severe, chronic low back pain with a neuropathic component: a randomized, double-blind, phase 3B study. Pain Pract. 2015;15(5):455-470.
35. Baron R, Kern U, Müller M, Dubois C, Falke D, Steigerwald I. Effectiveness and tolerability of a moderate dose of tapentadol prolonged release for managing severe, chronic low back pain with a neuropathic component: an open-label continuation arm of a randomized phase $3 \mathrm{~B}$ study. Pain Pract. 2015;15(5):471-486.

36. Baron R, Likar R, Martin-Mola E, et al. Effectiveness of tapentadol prolonged release (PR) compared with oxycodone/naloxone PR for the management of severe chronic low back pain with a neuropathic component: a randomized, controlled, open-label, phase $3 \mathrm{~b} / 4$ study. Pain Pract. 2016;16(5):580-599.

37. Ueberall MA, Mueller-Schwefe GH. Efficacy and tolerability balance of oxycodone/naloxone and tapentadol in chronic low back pain with a neuropathic component: a blinded end point analysis of randomly selected routine data from 12-week prospective open-label observations. J Pain Res. 2016;9:1001-1020.

38. Baron R, Kennes LN, Elling C. Retrospective analyses versus RCTs: comparing like with like? J Pain Res. 2017;10:783-786.

39. Guillén-Astete CA, Cardona-Carballo $\mathrm{C}$, de La Casa-Resino C. Tapentadol versus tramadol in the management of low back pain in the emergency department: impact of use on the need for reassessments. Medicine. 2017;96(45):e8403.

40. Notaro P. Tapentadol prolonged release in patients with severe chronic low back pain: results from a prospective, observational single-center study. Minerva Ortopedica e Traumatologica. 2017;68(1):13-19.

41. Finco G, Mura P, Musu M, et al. Long-term, prolonged-release oral tapentadol for the treatment of refractory chronic low back pain: a single-center, observational study. Minerva Med. 2018;109(4): 259-265.

42. Reimer M, Hüllemann P, Hukauf M, et al. Prediction of response to tapentadol in chronic low back pain. Eur J Pain. 2017;21(2):322-333.

43. Passavanti MB, Fiore M, Sansone P, et al. The beneficial use of ultramicronized palmitoylethanolamide as add-on therapy to tapentadol in the treatment of low back pain: a pilot study comparing prospective and retrospective observational arms. BMC Anesthesiol. 2017;17(1):171.

44. Teichtahl AJ, Mccoll G. An approach to neck pain for the family physician. Aust Fam Physician. 2013;42(11):774-777.

45. Langford RM, Knaggs R, Farquhar-Smith P, Dickenson AH. Is tapentadol different from classical opioids? A review of the evidence. $\mathrm{Br} J$ Pain. 2016;10(4):217-221.

46. Pergolizzi JV, Lequang JA, Taylor R, Ossipov MH, Colucci D, Raffa RB. Designing safer analgesics: a focus on $\mu$-opioid receptor pathways. Expert Opin Drug Discov. 2018;13(10):965-972.

47. Eichenbaum G, Göhler K, Etropolski M, et al. Does tapentadol affect sex hormone concentrations differently from morphine and oxycodone? An initial assessment and possible implications for opioid-induced androgen deficiency. J Opioid Manag. 2015;11(3):211-227.

48. Dart RC, Cicero TJ, Surratt HL, Rosenblum A, Bartelson BB, Adams EH. Assessment of the abuse of tapentadol immediate release: the first 24 months. J Opioid Manag. 2012;8(6):395-402.

49. Butler SF, Mcnaughton EC, Black RA. Tapentadol abuse potential: a postmarketing evaluation using a sample of individuals evaluated for substance abuse treatment. Pain Med. 2015;16(1):119-130.
Journal of Pain Research

\section{Publish your work in this journal}

The Journal of Pain Research is an international, peer reviewed, open access, online journal that welcomes laboratory and clinical findings in the fields of pain research and the prevention and management of pain. Original research, reviews, symposium reports, hypothesis formation and commentaries are all considered for publication.

\section{Dovepress}

The manuscript management system is completely online and includes a very quick and fair peer-review system, which is all easy to use. Visit http://www.dovepress.com/testimonials.php to read real quotes from published authors. 